\title{
Höhere Anforderungen in der beruflichen Erstausbildung?
}

Mit einem Hauptschulabschluss haben Schulabgänger heute nur noch einen eingeschränkten Zugang zum Ausbildungsmarkt. Häufig müssen sie einen Umweg über das sogenannte Übergangssystem in Kauf nehmen oder eine Ausbildung in einem wenig beschäftigungssicheren Berufssegment beginnen. Liegt das daran, dass die Anforderungen in der beruflichen Erstausbildung im Zuge der Bildungsexpansion sowie des technologischen und strukturellen Wandels gestiegen sind? Erste empirische Anhaltspunkte zur Beantwortung dieser Frage liefert die historisch-vergleichende Auswertung der Ausbildungsordnungen ausgewählter Berufe. ${ }^{\circ}$

PAULA PROTSCH

\section{Einleitung}

Hauptschülerinnen und Hauptschüler ${ }^{2}$ in Deutschland haben heutzutage vergleichsweise geringe Chancen, im Anschluss an die Schule eine vollqualifizierende Erstausbildung zu beginnen. Sie besuchen häufiger als höher gebildete Schulabgänger eine Bildungsmaßnahme im sogenannten Übergangssystem und finden nur innerhalb eines begrenzten und weniger attraktiven Berufsspektrums einen Ausbildungsplatz (Autorengruppe Bildungsberichterstattung 2012, S. 110ff.). Es ist also nicht nur die bekannte Segregation nach Geschlecht, sondern auch eine berufliche Segmentierung nach Bildungsgruppen zu beobachten, die zu ungleichen Bedingungen bei der Berufsfindung und damit verbunden zu geringeren Erwerbsaussichten führt.

$\mathrm{Ob}$ die geringen Ausbildungschancen von Hauptschülern auf steigende berufliche Anforderungen zurückzuführen sind, wird vielfach diskutiert (siehe z. B. Kleinert/Jacob 2012; Uhly/Erbe 2007). Mangels entsprechender empirischer Analysen sind mögliche Zusammenhänge bisher jedoch unbekannt. Für eine zielführende Diskussion und für gelingende Interventionen zur Unterstützung von Übergängen in eine Ausbildung ist es jedoch wichtig zu wissen, ob beobachtbare Anhebungen der rechtlichen oder praktizierten schulischen Zugangsvoraussetzungen tatsächlich darauf zurückzuführen sind, dass es heutzutage grundsätzlich schwieriger geworden ist, einen Ausbildungsberuf zu erlernen. Mittels einer historisch vergleichenden, qualitativen Inhaltsanalyse der Ausbildungsordnungen (VO) ausgewählter dualer Ausbildungsberufe wird in diesem Beitrag daher untersucht, inwiefern sich erhöhte Anforderungen in der beruflichen Erstausbildung beobachten lassen, die für verringerte Ausbildungschancen von Hauptschülern ursächlich sein könnten.

\section{Hintergrund}

Seit den 1950er Jahren haben sich die Ausbildungschancen von Jugendlichen, die höchstens über einen Haupt-

Die Analysen für diesen Beitrag entstanden im Rahmen des Projekts "The ,Discovery' of Youth's Learning Potential Early in the Life Course" am Wissenschaftszentrum Berlin für Sozialforschung (WZB). Mein Dank gilt der Jacobs Foundation für die Förderung des Projekts.

(2) Alle männlichen Bezeichnungen von Personen und Berufen beziehen sich im Folgenden auf beide Geschlechter. Als Hauptschüler werden in diesem Beitrag alle Jugendlichen bezeichnet, die die allgemeinbildende Schule ohne Hauptschulabschluss bzw. mit Erreichen eines Hauptschulabschlusses verlassen haben - unabhängig vom tatsächlich besuchten Schultyp. 
schulabschluss verfügen, zunehmend auf weniger attraktive Berufssegmente konzentriert, in denen eine Ausbildung mit einem höheren Arbeitslosigkeitsrisiko im späteren Erwerbsverlauf einhergeht. Dies zeigen quantitative Analysen auf Basis der Deutschen Lebensverlaufsstudien des MaxPlanck-Instituts für Bildungsforschung und der Befragung „Arbeiten und Lernen im Wandel" des Instituts für Arbeitsmarkt- und Berufsforschung (Protsch 2012). Es handelt sich dabei um Segmente, die Bau-, Gastronomie-, Verkaufs- und Metallberufe umfassen. Diese Analysen zeigen auch, dass verringerte Ausbildungschancen der Hauptschüler in attraktiveren Berufssegmenten - dazu zählen das Elektro-, Gesundheits- und Bürosegment - nicht durch rein kapazitätsabhängige Verdrängungsprozesse verursacht werden, die sich bei einer ausgeglichenen Angebots- und Nachfragerelation auf dem Ausbildungsmarkt wieder verbessern würden. Bekannt ist auch, dass die sich historisch verschlechternden Chancen dieser Bildungsgruppe nicht allein auf eine - im Zuge der Bildungsexpansion - sozial selektiver werdende Zusammensetzung der Hauptschülerschaft zurückzuführen sind (Kleinert/Jacob 2012). Welche anderen Erklärungen lassen sich anführen?

Neben den ausbildenden Betrieben und Schulen gestaltet eine Vielzahl weiterer Akteure das Geschehen auf dem Ausbildungsmarkt. Gewerkschaften, Arbeitgeberorganisationen, aber auch Bund und Länder sowie das Bundesinstitut für Berufsbildung (BIBB) sind maßgeblich beteiligt. Die beruflichen Akteure können als Gatekeeper („Torhüter“) bezeichnet werden. Sie zählen zu den Entscheidungsträgern bei einem wichtigen Übergang im Lebensverlauf - dem Übergang von der Schule in den Arbeitsmarkt (Struck 2001). Berufliches Gatekeeping findet in Deutschland nicht nur auf der betrieblichen Ebene - beim tatsächlichen Rekrutierungs- und Einstellungsprozess - statt, sondern auch auf der regulativen Ebene der beruflichen Bildung. Ein prominentes Beispiel für Letzteres ist die Neuordnung von Ausbildungsberufen im dualen System, bei der - unter der Beteiligung der oben genannten Akteure - bundesweit gültige Mindestvorgaben für die Durchführung sowie die Inhalte einer Berufsausbildung entwickelt und festgelegt werden. In Anlehnung an Meyer (1977) kann das Gatekeeping auf dem Ausbildungsmarkt als eine Institutionalisierung von (1) Wissensstandards in Bezug auf die Inhalte und die Komplexität des Anforderungsniveaus von Ausbildungsberufen und (2) Verhaltensnormen hinsichtlich gängiger Einstellungskriterien auf der betrieblichen Ebene verstanden werden.

Durch die Bildungsexpansion im Sekundarschulbereich ist seit den 1950er Jahren der Hauptschüleranteil an den Schülern insgesamt von fast $80 \%$ auf ca. $20 \%$ gesunken (vgl. Geißler 2006, S. 275). Höher qualifizierte Schulabgänger standen damit dem Ausbildungsmarkt im Verlauf der letzten Jahrzehnte in einem deutlich größeren und wachsenden Umfang zur Verfügung, sodass Betriebe vermehrt Jugendliche mit Realschulabschluss oder Abitur auswählen konnten. Abgesehen von der qualifikatorischen Verschie- bung der Schulabgängerstruktur und demografischen Veränderungsprozessen, können berufliche Gatekeeper die schulischen Voraussetzungen für den Zugang zur Berufsausbildung auch in Reaktion auf wirtschaftliche und technologische Entwicklungen angehoben haben, die zu einer Steigerung der beruflichen Anforderungen beigetragen haben könnten (siehe z. B. Baker 2009).

\section{Veränderung und Kontinuität des Anspruchsniveaus in der beruflichen Erstausbildung}

Ist es heute schwieriger geworden, einen vollqualifizierenden Ausbildungsberuf zu erlernen? Im Folgenden wird die (kognitive) Komplexität der zu vermittelnden beruflichen Kenntnisse und Fähigkeiten in ausgewählten dualen Ausbildungsberufen sowie deren Veränderung im historischen Vergleich untersucht. Inhaltsanalytisch werte ich die Ausbildungsrahmenpläne für den betrieblichen Teil einer Berufsausbildung aus, die ein Bestandteil der Ausbildungsordnungen sind. Die Lernziele in den Rahmenplänen sollen während einer Ausbildungsdauer von drei und in einigen Berufen auch dreieinhalb Jahren erreicht werden.

Seit dem ersten Berufsbildungsgesetz (BBiG), das 1969 in Kraft trat, treffen bei der Neuordnung von Ausbildungsberufen des dualen Systems Repräsentanten von Arbeitgeberverbänden, Gewerkschaften, Bund und Ländern sowie Berufsbildungsexperten des BIBB zusammen. ${ }^{3}$ Zwischen den verschiedenen Interessenvertretern wird dann - oftmals über einen Zeitraum von mehreren Jahren - nach dem Konsensprinzip über Neuordnungen von Ausbildungsberufen verhandelt. Mit anderen Worten: Ausbildungsordnungen werden in der Regel nur dann vom zuständigen Bundesministerium erlassen, wenn alle beteiligten Sozialpartner zugestimmt haben (BiBB 2011). ${ }^{\circledR}$

Im Rahmen eines Neuordnungsverfahrens können der Zugang zu und das Erlernen von Ausbildungsberufen im

(3) Die Bundesländer und die Kultusministerkonferenz sind für den Unterricht an den Berufsschulen zuständig. Die Rahmenlehrpläne für den Berufsschulunterricht haben sehr viel weniger bindenden Charakter als die Ausbildungsordnungen, die der Bund erlässt. Im Handwerk werden Ausbildungsordnungen nicht direkt auf der Grundlage des BBiG, sondern auf Basis der Handwerksordnung ( $\mathrm{HwO}$ ) erlassen. Diese entspricht an den betreffenden Stellen jedoch dem BBiG.

(4) Das Konsensprinzip wurde Anfang der 2000er Jahre verletzt, als die Bundesregierung Ausbildungsordnungen zu neuen oder modernisierten zweijährigen Ausbildungsberufen erlassen hat - gegen die strikte Weigerung der Gewerkschaften (Kath 2005). 
ÜBERSICHT 1

\section{Betrachtete Ausbildungsberufe (Fallbeispiele)}

Berufssegment Berufsbezeichnung

Ausbildungsordnungen nach Berufsbildungsgesetz/Handwerksordnung

Ausbildungsberufe mit relativ guten Ausbildungschancen für Hauptschüler, aber geringeren Beschäftigungsperspektiven nach erfolgreichem Abschluss

\begin{tabular}{|c|c|c|}
\hline Bau & $\begin{array}{l}\text { Maler und Lackierer } \\
\text { Fachrichtung: Gestaltung und Instandhaltung }\end{array}$ & $\begin{array}{l}\text { VO } 1975 \\
\text { VO } 2003\end{array}$ \\
\hline Gastronomie & Koch & $\begin{array}{l}\text { VO } 1979 \\
\text { VO } 1998\end{array}$ \\
\hline Metall & $\begin{array}{l}\text { Metallbauer } \\
\text { Fachrichtung: Konstruktionstechnik } \\
\text { Vorgängerberuf: Schlosser und Schmied bis } 1989\end{array}$ & $\begin{array}{l}\text { VO } 1989 \\
\text { VO } 2002 / 2008\end{array}$ \\
\hline & $\begin{array}{l}\text { Kraftfahrzeugmechatroniker } \\
\text { Fachrichtung: Personenkraftwagentechnik } \\
\text { Vorgängerberuf: Kraftfahrzeugmechaniker bis } 2003\end{array}$ & $\begin{array}{l}\text { VO } 1973 \\
\text { VO } 1989 \\
\text { VO } 2003 / 2007\end{array}$ \\
\hline Verkauf & Kaufmann im Einzelhandel & $\begin{array}{l}\text { VO } 1987 \\
\text { VO } 2004 / 2009\end{array}$ \\
\hline & $\begin{array}{l}\text { Kaufmann im Groß- und Außenhandel } \\
\text { Fachrichtung: Außenhandel }\end{array}$ & $\begin{array}{l}\text { VO } 1973 \\
\text { VO } 1978 \\
\text { VO } 1997 \\
\text { VO } 2006\end{array}$ \\
\hline
\end{tabular}

Ausbildungsberufe mit relativ schlechten Ausbildungschancen für Hauptschüler, aber günstigen Beschäftigungsperspektiven nach erfolgreichem Abschluss

\begin{tabular}{l|l|l}
\hline \multirow{2}{*}{ Elektro } & $\begin{array}{l}\text { Elektroniker } \\
\text { Fachrichtung: Energie- u. Gebäudetechnik (Handwerk) } \\
\text { Vorgängerberuf: Elektroinstallateur bis 1987 }\end{array}$ & VO 1987 \\
& $\begin{array}{l}\text { Medizinischer Fachangestellter } \\
\text { Vorgängerberuf: Sprechstundenhelfer bis 1985/ Arzt- } \\
\text { helfer bis 2006 }\end{array}$ & VO 1985 \\
\hline Gesundheit & $\begin{array}{l}\text { Bürokaufmann } 2006 \\
\text { Küro }\end{array}$ & VO 1991 \\
& Fachangestellter für Bürokommunikation & VO 1991 \\
\hline
\end{tabular}

Quelle: Zusammenstellung der Autorin.

dualen System erleichtert oder erschwert werden. Anzumerken ist jedoch, dass die rechtlichen Zugangsvoraussetzungen in Bezug auf die schulische Vorbildung der Auszubildenden vonseiten der beruflichen Gatekeeper nicht verändert werden können. Ausbildungen im dualen System setzen, im Gegensatz zu Ausbildungsberufen im Schulberufssystem, formal keinen (spezifischen) Schulabschluss voraus. Das schließt jedoch keineswegs aus, dass die Arbeitgeber als die betrieblichen Gatekeeper einen bestimmten Schulabschluss als Einstellungsvoraussetzung für eine Ausbildung in ihrem Unternehmen festlegen können.

Ausbildungsordnungen für duale Ausbildungsberufe können aber hinsichtlich der Inhalte, des Anforderungsniveaus und der Komplexität der beruflichen Kenntnisse sowie der Ausbildungsdauer verändert werden. Damit steht für Betriebe eine bundesweit anzuwendende Rechtsnorm über gültige Wissensstandards bereit, die nicht nur während der Ausbildung zum Tragen kommt, sondern auch eine Orientierungshilfe und Legitimationsbasis bei der Auswahl der Auszubildenden bieten kann.

\subsection{Betrachtete Ausbildungsberufe}

Die Bezeichnungen der betrachteten Ausbildungsberufe, ihrer Vorgängerberufe und der analysierten Dokumente (der Ausbildungsordnungen) finden sich in Übersicht $1 .^{\oplus}$ Der Beobachtungszeitraum der Untersuchung beginnt mit den ersten Ausbildungsordnungen, die für die betrach-

5 Das BIBB stellt auf seiner Website Informationen über die Entstehungsgeschichte der einzelnen Ausbildungsberufe und ihrer Bezeichnungen zur Verfügung, vgl. http://www2. bibb.de/tools/aab/aabberufeliste.php. 
teten Ausbildungsberufe nach dem BBiG von 1969 bzw. der Handwerksordnung ( $\mathrm{HwO}$ ) erlassen wurden, und endet mit den jeweils aktuellen VO. Erst seit 1969 wurden nach und nach standardisierte Ausbildungsrahmenpläne entwickelt, die einen Vergleich der zu vermittelnden Lernziele zulassen. Die Verordnungsaktivität für einen Ausbildungsberuf, das heißt die Häufigkeit einer Neuordnung und die Geltungsdauer der jeweiligen VO, wird in der Auswertung als ein erster Indikator für das Ausmaß der Veränderungen innerhalb eines Berufs verwendet.

Die Auswahl der Fallbeispiele war davon geleitet, für jedes quantitativ relevante Berufssegment des Ausbildungsmarktes einen Ausbildungsberuf zu identifizieren, in dem sowohl heute als auch in früheren Jahren ein vergleichsweise hoher Anteil an Jugendlichen ausgebildet wurde. Für eher heterogene Berufssegmente wurden jeweils zwei Berufe ausgewählt. Alle Fallbeispiele befinden sich schon seit mehreren Jahren unter den am stärksten besetzten Ausbildungsberufen von männlichen bzw. weiblichen Jugendlichen (BMBF 2011).

Die Ausbildungsberufe sind grob danach differenziert, ob sie Berufssegmenten angehören, die eher günstige oder eher nachteilige Beschäftigungsperspektiven bieten. In den attraktiveren Berufssegmenten, die sich durch günstige Beschäftigungsperspektiven auszeichnen (Elektro-, Gesundheits- und Bürosegment), haben sich die Chancen der Hauptschüler auf einen Ausbildungsplatz in den letzten Jahren merklich verringert, während sie in den weniger attraktiven Berufssegmenten (Bau-, Gastronomie-, Metallund Verkaufssegment) auch heute noch relativ gut sind. Nicht betrachtet werden Ausbildungsberufe, wie zum Beispiel Bank- oder Immobilienkaufmann, in denen Hauptschüler bereits in den 1960er und 1970er Jahren nur geringfügig ausgebildet wurden.

\subsection{Methodisches Vorgehen}

Aufbauend auf der Taxonomie der Lernziele im kognitiven Bereich nach Benjamin Bloom leite ich theoretisch ein Klassifikationssystem her (Anderson/Krathwohl 2001; Bloom 1956). Dieses Klassifikationssystem entwickele ich im Rahmen einer qualitativen Inhaltsanalyse nach Mayring (2008) weiter und wende es auf alle Fallbeispiele an. Die Lernziele in den Rahmenplänen benennen Kenntnisse oder Fähigkeiten, die während der Ausbildung zu erlernen sind; sie weisen auf das Komplexitätsniveau, auf dem dies geschehen soll, hin und sind zudem zeitlich gegliedert. Für jedes Lernziel ist vermerkt, wie viele Wochen für das Erreichen des Lernziels vorzusehen sind. Dies erlaubt, die Lernziele in den Ausbildungsrahmenplänen einerseits hinsichtlich ihrer kognitiven Komplexität zu codieren und andererseits mit einem Faktor zu versehen, der dem vorgesehenen Zeitanteil in Wochen - an der Ausbildungszeit insgesamt - entspricht. Auf diese Weise kann berechnet werden, wie hoch der prozentuale Anteil einer bestimmten Art von Lernzielen an der Ausbildungsdauer insgesamt ist. ${ }^{\circ}$
Dieses Vorgehen ermöglicht darüber hinaus zu beobachten, wie sich die Anteile verschiedener Lernziele innerhalb eines Ausbildungsberufs über die Zeit verändert haben. Innerhalb eines Berufs wird die Zunahme des Anteils kognitiv komplexerer Lernziele über die Berechnung der Differenz der entsprechenden Kategorien von zwei aufeinanderfolgenden VO in Prozentpunkten berechnet. Ist beispielsweise in einem Beruf der Anteil der Lernziele in einer Kategorie $\mathrm{x}$, die als kognitiv komplexer gilt als die Kategorien y und z, im Zeitverlauf - von einer VO zur nächsten VO - um 30 Prozentpunkte gestiegen, ist dies als eine Erhöhung des Anforderungsniveaus in diesem Beruf zu bewerten. Im Gegensatz dazu wäre das Ausmaß der Veränderung entsprechend geringer einzustufen, wenn der Anteil kognitiv komplexerer Lernziele lediglich um 10 oder 15 Prozentpunkte gestiegen wäre.

Der Taxonomie nach Bloom folgend werden über Lernziele die Kenntnisse und Fähigkeiten formuliert, die zu erlernen unterschiedlich komplex sein kann. Das heißt, sie erfordern ungleich anspruchsvolle kognitive Prozesse. Diese Prozesse, deren Schwierigkeitsgrad entlang eines Kontinuums ansteigt, werden bezeichnet als: Erinnern, Verstehen, Anwenden, Analysieren, Beurteilen und (etwas Neues) Erschaffen. Durch den jeweils zugrunde liegenden kognitiven Prozess eignen sich Individuen den Inhalt der Lernziele an. In der vorliegenden Analyse werden die Lernziele in erster Linie hinsichtlich ihrer kognitiven Komplexität klassifiziert, weniger hinsichtlich der spezifischen Inhalte. Auf einer vertikalen Dimension werden daher zwei bis vier Schwierigkeitsgrade abgegrenzt, die für das Niveau des kognitiven Prozesses stehen, auf dem die zu kategorisierenden Lernziele erlernt werden sollten.

Gleichwohl unterscheidet das verwendete Klassifikationssystem auf einer horizontalen Dimension die Wissenskategorien „Theoretisches Vorwissen“, „Berufliches Handeln“, „Interaktion mit Kunden/Patienten“ sowie „Fremdsprachenkenntnisse“. Während einer Berufsausbildung werden sowohl theoretische Kenntnisse als auch praktische Fähigkeiten erlernt. Lernziele, die der Wissenskategorie „Theoretisches Vorwissen“ zuzuweisen sind, sind nicht im Sinne von akademisch-theoretischem Wissen zu verstehen, sondern als ein Vorwissen, das eine Voraussetzung für das berufliche Handeln darstellt. „Theoretisches Vorwissen“ ist daher innerhalb eines Berufs immer als Vorstufe für das anwendungsbezogene berufliche Handeln zu verstehen und in der Regel weniger kognitiv komplex als Lernziele, die als „Berufliches Handeln“ klassifiziert werden. Damit wird das Klassifikationssystem der stark anwendungsbezogenen Orientierung der Berufsausbildung gerecht. Lassen sich ein Anstieg des Anteils der Lernziele in der Wissenskategorie „Berufliches Handeln“ be-

6 Für einen ähnlichen Ansatz zur Untersuchung der Kompetenzorientierung in aktuellen Ausbildungsordnungen unterschiedlicher Ausbildungsberufe siehe Hensge et al. 2009; Schreiber 2009. 
Die kognitive Komplexität der Lernziele in Ausbildungsordnungen dualer Ausbildungsberufe (Klassifikationssystem)

\begin{tabular}{|c|c|c|c|c|}
\hline \multirow{2}{*}{ Wissenskategorie } & \multicolumn{2}{|c|}{ Grundlegendes berufliches Wissen } & \multicolumn{2}{|l|}{ Besondere berufliche Kenntnisse } \\
\hline & $\begin{array}{l}\text { Theoretisches } \\
\text { Vorwissen }\end{array}$ & Berufliches Handeln & $\begin{array}{l}\text { Interaktion mit } \\
\text { Kunden oder Patienten }\end{array}$ & Fremdsprachen \\
\hline \multirow{6}{*}{$\begin{array}{l}\text { Kognitiver Prozess } \\
\text { (Schwierigkeitsgrad) }\end{array}$} & $\begin{array}{l}\text { a) Erinnern } \\
\text { (von Faktenwissen) }\end{array}$ & A) Anwenden unter Anleitung & $\begin{array}{l}\text { la) Einfachere Interaktion } \\
\text { (Anwenden) }\end{array}$ & $\begin{array}{l}\text { Ila) Grundkenntnisse } \\
\text { (Erinnern) }\end{array}$ \\
\hline & $\begin{array}{l}\text { z. B. etwas nennen, erkennen, } \\
\text { aufzählen }\end{array}$ & $\begin{array}{l}\text { z. B. alle Tätigkeiten unter/nach } \\
\text { Anleitung/Anweisung }\end{array}$ & $\begin{array}{l}\text { z. B. Kunden oder } \\
\text { Patienten informieren, betreuen }\end{array}$ & $\begin{array}{l}\text { z. B. fremdsprachige/englische } \\
\text { Fachausdrücke anwenden }\end{array}$ \\
\hline & $\begin{array}{l}\text { b) Verstehen (von Zusammen- } \\
\text { hängen, Sachverhalten) } \\
\text { z .B. etwas erklären, erläutern, } \\
\text { verstehen, zuordnen }\end{array}$ & $\begin{array}{l}\text { B) Selbstständiges (einfache- } \\
\text { res) Anwenden } \\
\text { z. B. Regeln, Grundsätze, Ver- } \\
\text { fahren anwenden, etwas bear- } \\
\text { beiten, durchführen }\end{array}$ & \multirow{4}{*}{$\begin{array}{l}\text { Ib) Komplexere Interaktion } \\
\text { (komplexes Anwenden, } \\
\text { erforderlich: Analysieren und/ } \\
\text { oder Bewerten) } \\
\text { z. B. Kunden oder } \\
\text { Patienten beraten, motivieren, } \\
\text { etwas erklären, Verkaufsge- } \\
\text { spräch führen }\end{array}$} & \multirow{4}{*}{$\begin{array}{l}\text { Ilb) Erweiterte Kenntnisse } \\
\text { (Verstehen, Anwenden, Analy- } \\
\text { sieren etc.) } \\
\text { z. B. fremdsprachiges Fachge- } \\
\text { spräch möglich, } \\
\text { fremdsprachige Texte lesen, } \\
\text { auswerten }\end{array}$} \\
\hline & $\begin{array}{l}\text { c) Analysieren und/oder Bewer- } \\
\text { ten (von Zusammenhängen, } \\
\text { komplexen Sachverhalten) }\end{array}$ & $\begin{array}{l}\text { C) Selbstständiges komplexes } \\
\text { Anwenden (erforderlich: Analy- } \\
\text { sieren und/oder Bewerten inte- } \\
\text { griert im Anwenden) }\end{array}$ & & \\
\hline & $\begin{array}{l}\text { z. B. etwas (theoretisch) be- } \\
\text { rechnen, begründen, abschät- }\end{array}$ & $\begin{array}{l}\text { z. B. etwas kontrollieren, orga- } \\
\text { nisieren, prüfen, planen }\end{array}$ & & \\
\hline & $\begin{array}{l}\text { (Anmerkung: Diese Kategorie } \\
\text { traf nur sehr selten. Sie betraf } \\
\text { maximal } 3 \% \text { der Ausbildungs- } \\
\text { zeit) }\end{array}$ & $\begin{array}{l}\text { D) Etwas Erschaffen/ } \\
\text { Innovatives Handeln } \\
\text { z. B. etwas entwerfen, eigene } \\
\text { Lösungen entwickeln, eigene } \\
\text { Vorschläge machen }\end{array}$ & & \\
\hline
\end{tabular}

Quelle: Zusammenstellung der Autorin

obachten und eine gleichzeitige Abnahme des Anteils der Lernziele in der Kategorie „Theoretisches Vorwissen“, so ist davon auszugehen, dass das Erlernen eines Ausbildungsberufs kognitiv komplexere Lernprozesse erfordert und damit schwieriger geworden ist. Gleichermaßen ist ein gestiegenes Anforderungsniveau festzustellen, wenn beispielsweise in den Ausbildungsordnungen eines Berufs innerhalb der Wissenskategorie „Berufliches Handeln“ eine Verschiebung hin zu höheren Schwierigkeitsgraden stattgefunden hat. Dies wäre der Fall, wenn im Vergleich zu einer älteren VO gemäß der aktuellen VO deutlich mehr Zeit der Vermittlung von Lernzielen gewidmet werden soll, die unter die Schwierigkeitsgrade „C) selbstständiges komplexes Anwenden“ oder „D) etwas Erschaffen/Innovatives Handeln“ fallen.

Neben diesen beiden allgemeinen Wissenskategorien wurden zwei weitere Kategorien mit spezifischeren Lernzielen definiert. Es handelt sich dabei um die „Interaktion mit Kunden/Patienten“, die zu großen Teilen soziale Kompetenzen umfasst, und um „Fremdsprachenkenntnisse“. Diese beiden Wissenskategorien enthalten somit Fähigkeiten, deren Bedeutung für die berufliche Ausbildung und den Arbeitsmarkt heutzutage immer häufiger betont wird. In Bezug auf die Fremdsprachenkenntnisse, bei denen es sich überwiegend um Englischkenntnisse handelt, ist jedoch anzumerken, dass die Vermittlung von Fremdsprachenkenntnissen auch im allgemeinbildenden Schulsystem seit den 1960er Jahren an Bedeutung gewonnen hat. ${ }^{\circ}$ Das verwendete Klassifikationssystem ist Übersicht 2 zu entnehmen.

\subsection{Ergebnisse}

Die Auswertung der Ausbildungsrahmenpläne ist nach Beruf und Jahr der Ausbildungsordnung in Abbildung $1 \mathrm{zu}-$ sammengefasst. Die Balkendiagramme zeigen, welchen prozentualen Anteil die den einzelnen Lernzielen zugeordneten Wissenskategorien und Schwierigkeitsgrade jeweils an der Ausbildungsdauer der betrachteten Ausbildungsberufe insgesamt haben und welche Veränderungsprozesse diesbezüglich im Zeitverlauf beobachtbar sind.

Die Ergebnisse machen grundsätzlich deutlich, dass die Wissenskategorie „Interaktion mit Kunden/Patienten“

(7) Laut dem Hamburger Abkommen von 1964 wird bundesweit in allen Schulen ab Klasse 5 eine erste Fremdsprache, in der Regel Englisch, unterrichtet (KMK 1971). 
und in geringerem Ausmaß „Fremdsprachenkenntnisse“ in der Erstausbildung berufsübergreifend (zum Teil erstmalig) an Bedeutung gewonnen haben. Auszubildende müssen sich heute zu einem gewissen Teil andere Kenntnisse - darunter soziale Kompetenzen und Fremdsprachenkenntnisse - aneignen als Auszubildende in früheren Jahrzehnten. Zwischen den Berufen lassen sich jedoch merkliche Unterschiede feststellen. Während sich beispielsweise für den Metallbauer lediglich eine Zunahme dieser Kenntnisse um zwei Prozentpunkte festmachen lässt, beträgt der Anstieg für die Berufe Elektroniker oder Kaufmann im Groß- und Außenhandel 14 Prozentpunkte. Auch in diesen Ausbildungsberufen, in denen der Anteil der Lernziele in den „Besonderen Kenntnissen“ in einem substanziellen Ausmaß gestiegen ist, ist zudem nicht zwingend davon auszugehen, dass es schwieriger, das heißt kognitiv komplexer geworden ist, eine Ausbildung zu absolvieren. Stattdessen erfordert es heutzutage oftmals differenziertere Fähigkeiten.

\subsubsection{Berufe mit guten Chancen für Haupt- schüler, aber geringer Beschäftigungs- sicherheit}

$\mathrm{Zu}$ den betrachteten Ausbildungsberufen in Berufssegmenten mit auch heute noch vergleichsweise guten Zugangschancen für Hauptschüler gehören die Fallbeispiele Metallbauer (Metallsegment), Maler und Lackierer (Bausegment) und Koch (Gastronomiesegment). Diese Berufe weisen jeweils zwei VO seit dem BBiG von 1969 auf und eine geringe Zunahme der kognitiven Komplexität. Der Zeitanteil der Lernziele in den Ausbildungsrahmenplänen, die nun statt der Wissenskategorie „Theoretisches Vorwissen“ dem „Beruflichen Handeln“ angehören bzw. einer schwierigeren Unterkategorie innerhalb dieser beiden Wissenskategorien ist für den Beruf Maler und Lackierer um elf und für den Beruf Koch um 17 Prozentpunkte gestiegen. Der Ausbildungsrahmenplan der jüngsten VO des Berufs Metallbauer weist keine Zunahme kognitiv komplexerer Lernziele auf. Eine kleine Veränderung ist, wie bei den meisten Fallbeispielen, im Bereich der „Besonderen Kenntnisse“ zu beobachten. Der Zeitanteil der Lernziele in diesem Bereich beträgt nun $2 \%$ der Ausbildungszeit insgesamt. Zur Veranschaulichung: $2 \%$ der Ausbildungszeit entspricht bei einer Ausbildungsdauer von dreieinhalb Jahren einem Zeitraum von unter einer Woche. Anhand dieser drei Ausbildungsberufe lässt sich damit fast kein bzw. nur ein geringer Wandel der kognitiven Komplexität bzw. Differenziertheit der Lernziele feststellen.

Anders stellt sich die Entwicklung für weitere Fallbeispiele dar, die ebenfalls zu den weniger beschäftigungssicheren Berufssegmenten zu zählen sind. Dazu gehört der Beruf Kraftfahrzeugmechatroniker (zweites Fallbeispiel für das Metallsegment), für den einige Veränderungsprozesse zu beobachten sind. Hier fanden drei Verordnungsaktivitäten seit dem BBiG von 1969 statt. In der VO von 1973 beträgt der zeitliche Anteil der Lernziele, die der Wissenskategorie „Theoretisches Vorwissen“ angehören, noch fast 40 \%. In der
VO von 1989 ist diese Wissenskategorie kaum mehr vorhanden, während der Anteil der Lernziele in der Kategorie „Berufliches Handeln" deutlich gestiegen ist. Zu Anfang des Beobachtungszeitraums hat damit eine vergleichsweise hohe Zunahme der kognitiven Komplexität von 36 Prozentpunkten stattgefunden. Zwischen 1989 und 2003/2007 ist hingegen nur eine Veränderung von sieben Prozentpunkten in Richtung differenzierterer Fähigkeiten zu verzeichnen. Auszubildende in diesem Beruf werden heutzutage intensiver in der „Interaktion mit Kunden“ unterrichtet. ${ }^{8}$

Auch die Ausbildungsrahmenpläne der Fallbeispiele Kaufmann im Einzelhandel und insbesondere Kaufmann im Groß- und Außenhandel (beide zum Verkaufssegment zugehörig) weisen eine deutliche Zunahme der Anforderungen in der Erstausbildung auf. Zwar wurden für den Beruf Kaufmann im Einzelhandel wiederum nur zwei grundlegend überarbeitete Ausbildungsverordnungen seit dem BBiG von 1969 erlassen. Die Veränderung ist mit 36 Prozentpunkten jedoch vergleichsweise hoch. Die jüngste VO von 2004/2009 legt einen größeren Schwerpunkt auf die Wissenskategorien „Berufliches Handeln“ und „Interaktion mit Kunden“. Für das zweite Fallbeispiel des Verkaufssegments, den Kaufmann im Groß- und Außenhandel, sind die höchste Verordnungsaktivität und die größte Steigerung der kognitiv komplexeren Lernziele im Untersuchungssample nachzuvollziehen. Zudem ist eine relativ starke Zunahme im Lernbereich „Interaktion mit Kunden“ sowie „Fremdsprachenkenntnisse“ zu beobachten.

\subsubsection{Berufe mit geringen Chancen für Haupt- schüler, aber hoher Beschäftigungs- sicherheit}

Die Segmente der Elektro-, der Gesundheits- und der Büroberufe gehören zu den Berufssegmenten, in denen das Absolvieren einer Ausbildung eine höhere Beschäftigungssicherheit verspricht. Gleichzeitig sind im Zeitverlauf deutlich abnehmende Chancen für Hauptschüler zu beobachten. Der Ausbildungsberuf Elektroniker wurde in den Jahren 1987 und 2003 grundlegend neu geordnet. Für diesen Fall ist keine Steigerung des Vermittlungsanteils kognitiv komplexerer Lernziele auszumachen, sondern eine deutliche Verschiebung dieses Anteils in Richtung der „Besonderen Kenntnisse“. Der zeitliche Vermittlungsumfang der Wissenskategorie „Interaktion mit Kunden“ beträgt heute über $15 \%$ der Ausbildungszeit insgesamt und ist damit seit der VO

8 Die Ausbildungsordnungen von 2003 und 2007 werden zusammenfassend genannt, da der Ausbildungsrahmenplan von 2003 für die VO von 2007 nicht verändert wurde. Die Neuordnung erfolgte, da die Zwischenprüfung zum ersten Teil der Abschlussprüfung umgewandelt wurde. Diese Änderung wurde während der 2000er Jahre für viele andere duale Ausbildungsberufe ebenfalls vorgenommen. So auch für die Fallbeispiele Metallbauer, Kaufmann im Einzelhandel und Elektroniker. 
von 1987 um knapp neun Prozentpunkte gestiegen. Des Weiteren hat der Anteil der „Fremdsprachenkenntnisse“ um fast fünf Prozentpunkte zugenommen.

Nur der Ausbildungsberuf Medizinischer Fachangestellter (das Fallbeispiel für das Gesundheitssegment) weist gemäß der aktuellen VO von 2006 mit gut $24 \%$ Anteil an der Ausbildungszeit insgesamt eine noch größere Schwerpunktsetzung in der Kategorie „Besondere Kenntnisse“ auf. Die "Interaktion mit Patienten“ spielt in der Ausbildung eine große Rolle. Dies war bereits in der Ausbildung zum Vorgängerberuf Arzthelfer auf der Grundlage der VO von 1985 der Fall, wurde aber noch einmal um fast sieben Prozentpunkte erhöht. Darüber hinaus ist für diesen Beruf ein deutlicher Wandel hin zu kognitiv anspruchsvolleren Lernzielen festzustellen. Der prozentuale Anteil der Vermittlung von „Theoretischem Vorwissen“ wurde um 20 Prozentpunkte zugunsten des „Beruflichen Handelns“ reduziert.

Zu beachten ist, dass mit der VO von 1985 der zweijährige Anlernberuf Sprechstundenhelfer von dem regulär dreijährigen Ausbildungsberuf Arzthelfer abgelöst wurde. Mitte der 1980er Jahre fand außerdem ein weiterer Schritt in der Strukturierung der rechtlichen Rahmenbedingungen in den gesundheitsbezogenen Ausbildungsberufen statt. 1985 wurden erstmals für die auch zum Gesundheitssegment zählenden, aber dem Schulberufssystem zugehörigen Berufe in der Krankenpflege zeitliche und strukturelle Mindestangaben für die theoretische und praktische Ausbildung festgelegt (BA 2010, 2011).

Für das Gesundheitssegment sind damit wesentliche Steigerungen des Anspruchsniveaus in der Erstausbildung beobachtbar. Eine ähnliche Entwicklung lässt sich jedoch interessanterweise nicht für das Bürosegment nachweisen, obwohl in diesem Segment noch größere Einschnitte in den Zugangschancen von Hauptschülern im Zeitverlauf zu beobachten sind. Die Ausbildungsberufe Bürokaufmann, Kaufmann für Bürokommunikation sowie Fachangestellter für Bürokommunikation weisen unter den hier untersuchten Fallbeispielen die geringste Verordnungsaktivität auf. Seit dem BBiG von 1969 wurden erst- und bisher letztmalig in den Jahren 1991 bzw. 1992 Ausbildungsordnungen erlassen. Da es bisher keine Neuordnung für diese Berufe gab, ist nicht nachzuvollziehen, wie sich seitdem - in einem Zeitraum von 20 Jahren - die kognitive Komplexität der für diese Berufe zu erlernenden Fähigkeiten und Kenntnisse verändert hat. ${ }^{\ominus}$

\section{Schlussfolgerung}

Für Hauptschüler ist der Übergang in die Ausbildung zunehmend mit Schwierigkeiten behaftet. Nicht nur, dass sie häufig Umwege über das sogenannte Übergangssystem einschlagen müssen, ihre beruflichen Perspektiven beschränken sich zudem auf ein begrenztes Spektrum an Ausbildungsberufen, in denen Absolventen häufig mit einem hohen Ar-
ABB. 1

\section{Die kognitive Komplexität der Lernziele in den Ausbildungsrahmenplänen dualer Ausbildungsberufe}

Angaben in Prozent der Ausbildungszeit

[1. Theoretisches Vorwissen b)/c)

Berufliches Handeln B)

Theoretisches Vorwissen a)*

Berufliches Handeln A)

- Berufliches Handeln C)/D)

Interaktion mit Kunden oder Patienten la)/lb)

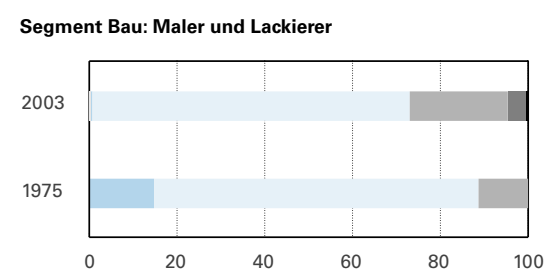

Segment Metall Fall 1: Metallbauer

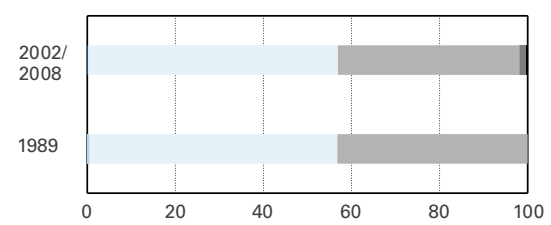

Segment Verkauf Fall 1: Kaufmann im Einzelhandel

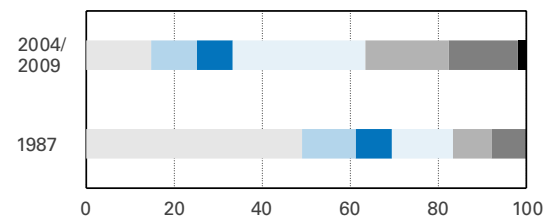

Segment Elektro: Elektroniker
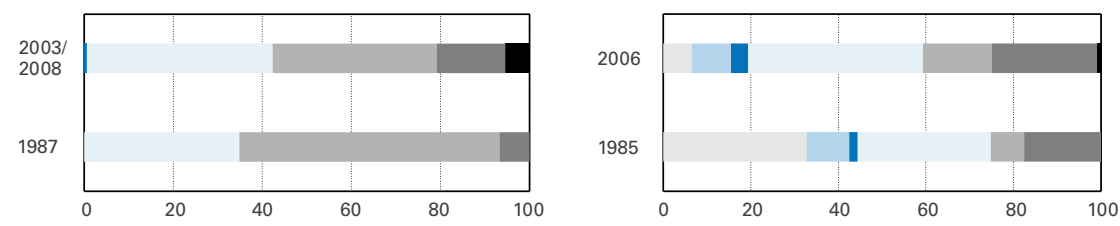

Segment Büro: Bürokaufmann

Sehr geringe Verordnungsaktivität. Die erste und bisher letzte VO nach dem BBiG wurde 1991 erlassen (das Gleiche gilt für die Berufe Kaufmann für Bürokommunikation (VO 1991) sowie Fachangestellter für Bürokommunikation (VO 1992)).

*Die Verweise a)-c), A)-D) und (a)-Ilb) beziehen sich auf das Klassifikationssystem aus Übersicht 2.

Quelle: Ausbildungsordnungen der Ausbildungsberufe aus verschiedenen Jahrgängen Bundesgesetzblatt; Analyse und Darstellung der Autorin.

beitslosigkeitsrisiko im weiteren Erwerbsverlauf rechnen müssen. Als eine populäre, wenn auch ungeprüfte, Er-

(9 Anzumerken ist, dass in einer kürzlich veröffentlichten Pressemitteilung des BIBB (2012) der Erlass einer Ausbildungsordnung für einen neuen Beruf mit der Bezeichnung „Kaufmann für Büromanagement" angekündigt wurde, der die drei separaten Ausbildungsberufe im Jahr 2013 oder 2014 aufheben soll. 
klärung für diese Entwicklung werden steigende Anforderungen in der Ausbildung genannt. Mit dieser Untersuchung unternehme ich anhand von Fallstudien einen ersten Versuch, empirische Anhaltspunkte für diese Debatte zu liefern.

Die Ergebnisse der historisch vergleichenden Inhaltsanalyse der Rahmenpläne von Ausbildungsordnungen dualer Ausbildungsberufe machen deutlich, dass von einem allgemeinen Trend kognitiv komplexer werdender Lernanforderungen nicht zu sprechen ist. Insofern ist auch keine allgemeingültige - in den rechtlichen Grundlagen der dualen Ausbildung fixierte Legitimationsbasis für eine Anhebung der schulischen Zugangsvoraussetzungen durch die betrieblichen Gatekeeper gegeben.

Damit ist nicht gesagt, dass die berufliche Erstausbildung in einigen der betrachteten Berufe nicht anspruchsvoller geworden ist, denn auch dafür gibt diese Untersuchung Hinweise. Deutliche Steigerungen der Anteile kognitiv komplexerer Lernziele und der Lernziele im Bereich der sozialen Kompetenzen sind zum einen für Ausbildungsberufe in Berufssegmenten festzustellen, in denen die Ausbildungschancen der Hauptschüler über die letzten Jahrzehnte deutlich gesunken sind. Dies zeigen die Fallbeispiele Medizinischer Fachangestellter und Elektroniker. Zum anderen lassen sich ebensolche Prozesse jedoch gleichzeitig für die Ausbildungsberufe Kaufmann im Einzelhandel, Kaufmann im Groß- und Außenhandel oder KFZMechatroniker beobachten, die zu den Berufssegmenten mit vergleichsweise guten Chancen für diese Bildungsgruppe zu zählen sind, die aber mit deutlich geringeren Beschäftigungsaussichten im Anschluss an die Ausbildung einhergehen.

Weder muss also ein erhöhtes Anforderungsniveau bedeuten, dass Hauptschüler keinen Ausbildungsplatz in den entsprechenden Berufen finden, noch sind dauerhaft angehobene schulische Voraussetzungen für attraktivere Ausbildungsberufe universell über komplexere Anforderungsniveaus erklärbar. Anzunehmen ist daher, dass andere Mechanismen für die abnehmenden Ausbildungschancen von Hauptschülern mitverantwortlich sein müssen. Eine ergänzende Erklärung bietet die Diskreditierungsthese von Solga (2005). Dieser These zufolge würden Hauptschüler im Zuge der Bildungsexpansion immer mehr als eine soziale Gruppe wahrgenommen, deren Bildungsanstrengung nicht ausreicht, um, wie die meisten anderen Schüler ihrer Geburtskohorten, einen mittleren oder höheren Schulabschluss zu erreichen. Diese Jugendlichen würden daher von betrieblichen Gatekeepern als „normabweichende Minderheit“ (ebd., S. 109) diskreditiert und nicht als Auszubildende akzeptiert - unabhängig davon, ob sich die beruflichen Anforderungen in der Erstausbildung erhöht haben oder nicht.
Baker, D. (2009): The educational transformation of work: towards a new synthesis, in: Journal of Education and Work 23 (3), S. 163-191

Bloom, B. S. (Hrsg.) (1956): Taxonomy of Educational Objectives: Handbook 1: Cognitive Domain, New York

Bundesagentur für Arbeit (BA) $(2010,2011)$ : BERUFENET Berufsinformationen einfach finden, http://berufenet.arbeitsagentur.de/berufe/search/simple/index. jsp (letzter Zugriff 11.10.2011)

Bundesinstitut für Berufsbildung (BIBB) (2011): Ausbildungsordnungen und wie sie entstehen, Schriftenreihe des Bundesinstituts für Berufsbildung, Bonn Bundesinstitut für Berufsbildung (BIBB) (2012): Aus Drei mach`Eins: Neuer Beruf „Kaufmann/Kauffrau für Büromanagement", Pressemitteilung des Bundesinstituts für Berufsbildung vom 07.03.2012, http://www.bibb.de/de/60894. htm (letzter Zugriff: 13.06.2012)

Bundesministerium für Bildung und Forschung (BMBF) (2011): Berufsbildungsbericht, Bonn

Geißler, R. (2006): Bildungsexpansion und Wandel der Bildungschancen. Veränderungen im Zusammenhang von Bildungssystem und Sozialstruktur, in: Ders. (Hrsg.): Die Sozialstruktur Deutschlands, Wiesbaden, S. 273-299

Hensge, K./Lorig, B./Schreiber, D. (2009): Kompetenzstandards in der Berufsausbildung, Bonn

Kath, F. (2005): Mehr Ausbildung durch verkürzte oder gestufte Ausbildungsberufe?, in: Berufsbildung in Wissenschaft und Praxis 34 (3), S. 5-8

Kleinert, C./Jacob, M. (2012): Strukturwandel des Übergangs in eine berufliche Ausbildung, in: Kölner Zeitschrift für Soziologie und Sozialpsychologie Sonderheft 52 (Soziologische Bildungsforschung), S. 211-233

Kultusministerkonferenz (KMK) (1971): Abkommen zwischen den Ländern der Bundesrepublik zur Vereinheitlichung auf dem Gebiete des Schulwesens, Abkommen vom 28.10.1964 in der Fassung vom 14.10.1971

Mayring, P. (2008): Qualitative Inhaltsanalyse - Grundlagen und Techniken, Weinheim/Basel

Meyer, J. W. (1977): The Effects of Education as an Institution, in: American Journal of Sociology 83 (1), S. 55-77

Protsch, P. (2012): Segmentierte Ausbildungsmärkte. Berufliche Chancen von Hauptschülerinnen und Hauptschülern im Wandel, Dissertationsschrift, Freie Universität Berlin

Schreiber, D. (2009): Wie kompetenzorientiert sind Ausbildungsordnungen eigentlich? Eine Ausbildungsordnungsanalyse, in: berufsbildung - Zeitschrift fü Praxis und Theorie in Betrieb und Schule 63 (116/117), S. 54-56

Solga, H. (2005): Ohne Abschluss in die Bildungsgesellschaft - Die Erwerbschancen gering qualifizierter Personen aus soziologischer und ökonomischer Perspektive, Opladen

Struck, O. (2001): Gatekeeping zwischen Individuum, Organisation und Institution. Zur Bedeutung und Analyse von Gatekeeping am Beispiel von Übergängen im Lebensverlauf, in: Leisering, L./Müller, R./Schumann, K. F. (Hrsg.): Institutionen und Lebensläufe im Wandel - Institutionelle Regulierungen von Lebensläufen, Weinheim/München, S. 29-54

Uhly, A./Erbe, J. (2007): Auszubildende mit Hauptschulabschluss - Vom Normalfall zur Randgruppe?, in: Berufsbildung in Wissenschaft und Praxis 36 (4), S. $15-20$

\section{LITERATUR}

Anderson, L./Krathwohl, D. R. (2001): A Taxonomy for Learning, Teaching and Assessing, New York

Autorengruppe Bildungsberichterstattung (2012): Bildung in Deutschland 2012. Ein indikatorengestützter Bericht mit einer Analyse zur kulturellen Bildung im Lebenslauf, Bielefeld

\section{AUTORIN}

PAULA PROTSCH, Sozialwissenschaftlerin, ist wissenschaftliche Mitarbeiterin in der Abteilung "Ausbildung und Arbeitsmarkt" am Wissenschaftszentrum Berlin für Sozialforschung (WZB). Arbeitsschwerpunkte: Bildung, Ausbildung, soziale Ungleichheit.

paula.protsch@wzb.eu 